\title{
Getting Free Heat Energy Based on Cavitation and Nuclear Fusion according to Revinov's Pilot Plants
}

\author{
Daurenbek Azenuly AUBAKIR
}

L. N. Gumilyov ENU, Astana, 010000, Kazakhstan

\begin{abstract}
In this report, the author describes and compares two innovative processes for producing thermal energy based on cavitation and nuclear fusion reactions in the indoor environment. Experiments conducted in the laboratory IHS (Intensive Heating System) of IE "Revinov N. M." indicate that the process of lasso-vortex cavitation, which generates by means of an electric arc obtained HV-EI, gas-liquid plasma state in the EHH-CTC (electro-hydraulic heater with cavitation thermal camera) is not inferior and sometimes even superior in heat transfer to NF-AC (nuclear fusion reactions in ambient conditions).
\end{abstract}

Key words: Free energy, lasso-vortex cavitation, electric arc, gas-liquid plasma, electro-hydraulic heater, cold nuclear fusion in ambient conditions.

\section{Introduction}

Since 2006, our initiative group of experts has been developing rapidly IHS (intensive heating systems) of different modifications: Lasso-vortex cavitation based on Ranke-Hilsch pipe, briefly called LVC-RHP; created in different years were called modifications $-\mathrm{VCH}$ (vortex cavitation heater), the $\mathrm{HCH}$ (hydrodynamic cavitation heater), sometimes referred to as HCR (hydrodynamic cavitation reactor); the current state of development is called the mini-thermal power plants based on the LVC-RHP to create IHS. As well as the development of IIHS (individually-intensive heating systems) on the basis of the electro-cavitation heater thermal camera, briefly called EHH-CTC, the current state of development is called a mini-boiler-based EHH-CTC to create IIHS. These developments gradually escalated into NF-RC (nuclear fusion reaction at room conditions). To us, all those seem to be natural.

\section{Natural Phenomena-the Mother of All the Above Processes}

The LVC-RHP process lasso-vortex cavitation in

Corresponding author: D. A. AUBAKIR, professor, research fields: mathematics and physics, cosmonautics and astronautics. the Ranke-Hilsch pipe has its progenitor-a tornado. Indeed, everything that happens inside RHP has been repeated in miniature of what takes place in the natural tornado: at one end of the fire spurts, the other end of hail pours. Similarly, during steady state, operation TPX inside the tube formed two parts, separated by a thin partition, on one side of which the water is more and more heated to a vapor state, and on the other side of the water it is still sensitive and sensitive cooled. However, it confirms the scientific assumption which J. Maxwell, Lord Kelvin sarcastically called "Maxwell's Demon". In a single vessel, all slower molecules can assemble at one end, while at the same time, the faster molecules rush to the other end. This is a fantasy, but a fact. Due to this phenomenon, LVC-RHP unit can be used as the heating and cooling apparatus. It has been realized by Soviet specialists Laboratory ONIL-9 in the Kuibyshev Aviation Institute for aviation and space technology.

The thermo-camera EHH-CTC process lasso-vortex cavitation in the gas-liquid plasma environment generated by an electric arc obtained HV-EI has its progenitor-lightning in miniature. Really, if lightning generates plasmids in the gas-drop environment cloud, the electric arc facilitates the formation of the 
gas-liquid plasma states in the electro-cavitation heater thermal camera.

In both cases, the installation produce more energy than they consume as food pump-in the case of the LVC-RHP, as a make-up HV-EI-in the case of EHH-CTC.

To describe this state of affairs, experts began to use an indicator of the ETC (energy transformation coefficient). Additional energy is also taken due to cavitation, i.e., the formation and collapse of countless air bubbles and their collapse because of high pressure in the caverns. By definition, the American experts, the temperature of the micro-explosions of bubbles varies from $5,000{ }^{\circ} \mathrm{C}$ to $25,000{ }^{\circ} \mathrm{C}$. Although these micro bursts last a few milliseconds, they manage to give impetus to their thermal environment, i.e., in this case-the water (in both cases, the working material is plain water at room temperature).

In fact, we are dealing with a supplement to the statement of V. I. Vernadsky that in nature a source of additional energy is radiation, and more cavitation has the same status, i.e., it is also a source of additional energy.

Turning now to nuclear fusion reactions in the indoor environment, we have to admit that as the natural ancestor we cannot with such clarity name one thing. There maybe a few "candidates", namely the transmutation of elements, natural radiation, glow, sonoluminescence, throw fire, radiation, thermonuclear reactions in stars and in the cores of planets and finally cavitation. However, the fact is that scientists not only cannot reach a consensus regarding the CNF-cold fusion, in our judgment, but think such a "spread" is the progenitor of this process. Psychologically, people in life is arranged so that the nature of the "do not run away, do not hide", used to live in analogy with natural phenomena, guided by the associative memory, genealogy whim, and here is the "spread of the broody-clocking hen!" That confused the children of Mother Nature, sometimes bringing the matter to the medieval Inquisition in modern guise, i.e.
To the "anti-scientific", "pseudo-scientific" and "Jesuitism", etc.

Some of these phenomena here are none other than the prototype of a prototype or, consequently, not the progenitor of CNF and with understanding of each of them is necessary to individuality, and at times it is necessary to look very deeply at the root. For example, transmutation, as applied to living organisms, what the authors examined thoroughly, led by renowned specialist V. I. Vysotsky [1].

We consider the concept closer to the same on the basis of what has been noted above that only the cavitation is produced by thermal energy from excess compared with the consumption of energy to feed on our established facilities, and there is a suspicion that the reaction of nuclear synthesis in the room conditions also cannot do without cavitation [2]. Even though we think that this phenomenon is the real progenitor of $\mathrm{CNF}$, it still requires a long time to be acknowledged. Looking ahead, we say that cavitation is the predecessor of the spontaneous phenomenon-the natural transmutation of animate and inanimate nature, the trump card in the effects of volcanic eruptions, the core symptoms and the magma of the Earth and other planets, the pair phenomena-thunder lightning, the main mechanism of thermal formation waters, dangerous weapon, wireworms cancer against its own enemy-the sea turtle, the main manifestations of solar activity in the form of granule formation and the same activities in the stars, and its role in the sequencing of many other natural phenomena is the main source of production of free energy, retarding spontaneous increase in entropy in the near-Earth space, i.e. it is indirectly responsible for the formation of the biosphere and other spheres, enveloping the Earth and protecting all life on the planet.

Along with such positive manifestations from cavitation, there are also negative ones as following: it corrodes the screws of ships and submarines, the blades of aircraft engines, particularly jet; it is responsible for in excess of the destructive 
manifestations of tornadoes, the nature and the cause of which is still not able to detect by any scientists or experts.

\section{3. "Rotational Energy" of Fominsky and Potapov's from Chrono-out!}

The appearance of this book is not to stop the orgy of indigestible official publication on science. On the contrary, it was neglected at all.

In the seventeenth chapter: "Heat generator of Potapov-cold fusion reactor" in "17.1. Cavitation as a substitute for a nuclear bomb," said [2, p. 317]:

"But in it there is something else that replaces an atomic bomb. Namely, the cavitation bubbles and cavities, resulting in water at the edge of the braking device of the vortex tube. Cavitation (from the Latin word cavitas-emptiness)-a discontinuity within the fluid, that is, education in her cavities filled with steam, gas, or a mixture thereof (the so-called cavitation bubbles). It arises as a result of local pressure reduction in the fluid below a critical value which is approximately equaled to the saturated vapor pressure of this liquid at this temperature. When pressure decrease occurs due to the local increase in the velocity of fluid flow (as in Potapov heat source), then this type called hydrodynamic cavitation. When pressure decrease occurs due to the passage of fluid into acoustic waves, it is called acoustic cavitation. According to existing ideas, most of cavitation bubbles almost immediately after they occur rapidly collapse under the influence of the ambient pressure. In this case they collapse at the end of a short time develop very high pressure gas mixture, filling the bubble and when the bubble adjacent to the solid surface, then there due to the collapse occurs microscopic jet stream of fluid that destroys the material of the surface. When the bubble is formed in an ultrasonic field under acoustic cavitation away from the solid surface, it may be quite a while, then periodically contracting then expanding, often at a frequency of ultrasonic vibrations, as noted in the paper by its Piersol. In the 30 years during acoustic cavitation investigations opened sonoluminescence. However, this phenomenon people were familiar before. Remember glowing in the dark surf astern motorboat-is sonoluminescence. But only glow for a long time Burun explained glow microorganisms disturbed screw boat. In practice it turned out that glow cavitation bubbles".

This moiety concept defined fully enough that cavitation is associated with a liquid medium, generally an aqueous medium, but not cavitation, and less active in the air. The first pioneering experiments were conducted by J. Ranke from 1931 to 1933, then the experiments by R. Hilsch in 1946 with the air. Although the statement that the air bubbles may appear in the air sounds unexpectedly and not accustomed to, this is so. It has also opened the eyes of Joseph Ranke and become his great merit.

Cavitation may also occur in other environments, particularly combined cycle, i.e. in the air-steam environment and in the air with snow and ice crystals that we have seen through a pair of lightning-bolt. However, this pair is no other than the sonoluminescence in the grandiose nature. To quote further [2, p. 318]:

"The founder of the theory of electrical sonoluminescence Frankel in 1940 suggested that the cavitation bubbles in the water appear in the same way as a crack in a solid. It is understandable-after all the water has a quasi crystal-like structure and because the water molecules are strongly polarized, at the opposite sides of the cracks in liquid water, according to Frankel opinion, there is significant charges of opposite sign, as in ionic crystals cracking. Then electrical discharges in gas-vapor medium begin to occur from the cavity between the walls, leading to excitation of molecules and atoms of the gas, followed by flashing of the photons".

At first, researchers thought that the electrification of the liquid in the cavitation bubbles is consistent with the well-known concepts Lenard that 
tribo-electric electrification liquid occurs only in violation of malice its surface and does not depend on the friction of liquid on the body of solid or gas, nor the nature of the gas, which contact liquid. Therefore, we assumed that after a cavitation bubble in the electric discharge occurs, the new charges there appear nowhere.

But experiments Gaitan and Krum have demonstrated that a single cavitation bubble without sharing continues to regularly emit more and more pulses of light over many cycles of expansion and contraction of it in an ultrasonic field. As in it, for such a short time between pulses recovers electrical charge required for the next discharge. Restored without disturbing the integrity of the surface of the bubble!

Failure to respond to this question has shaken the position of the electrical theory of sonoluminescence and forced many to return to the theory of heat. According to estimates of experts and based on the measured brightness, temperature of the gas in the bubble reaches the collapse of thousands and even millions of degrees, i.e. thermonuclear temperatures. Therefore, there are many hypotheses and speculations about the thermonuclear reactions supposedly going in cavitation bubbles and leading to the emission of their energy in much larger quantities than putting it in the water source of ultrasound, which provides the appearance of cavitation.

Further, with respect to the heat source Potapov said [2, p. 320]:

"At the same time, numerous calorimetric measurements carried out in different laboratories, have long shown that the energy yield of sonoluminescence (the ratio of the radiation energy to the energy deposited by ultrasound in water) is very low-less than the efficiency of the engine. High radiation appeared only the ratio of the instantaneous power to the power of ultrasound over 1,000. This indicated that cavitation only concentrated energy, and does not create additional. But the fact of the concentration of energy at first sight is contrary to the laws of thermodynamics, in particular the principle of increasing entropy".

In fact, it was much easier and more interesting. We have to have shown that in a vortex of water nuclear reaction between two protons, leading to the synthesis of ${ }^{2} \mathrm{D}$ deuteron, not twice banned and "normally permitted", as expressed by nuclear physicists, and can go with great speed.

To understand how and why this happens, let's fast forward to the nuclear reaction Eq. (1):

$$
{ }^{1} \mathrm{H}+{ }^{1} \mathrm{H} \rightarrow{ }^{2} \mathrm{D}+\mathrm{e}^{+}+v_{\mathrm{e}}+0.93 \mathrm{MeV}
$$

positrone $\mathrm{e}^{+}$symbol on the right side to the left:

$$
{ }^{1} \mathrm{H}+{ }^{1} \mathrm{H}+\mathrm{e}^{-} \rightarrow{ }^{2} \mathrm{D}+v_{\mathrm{e}}+1.953 \mathrm{MeV}
$$

Such a transfer is not prohibited by the rules "nuclear algebra" you only need to replace the portable particle antiparticle, in this case, the positron-electron.

Nuclear reaction (2), in principle, is also possible. Moreover, it has several advantages over a reaction (1) and the fact that the energy yield of the reaction increased to $1.95 \mathrm{MeV}$-not the most important of them. More importantly, it is now no longer have to wait long for the fluctuation of the weak (and therefore very slow) proton decay into a neutron, a positron and a neutrino, as it was in reaction (1), because now in the reaction (2) an electron is initially available in the ready-made and do not have to bear it. A process of changing the electron neutrino during a nuclear reaction is lighter than the process of the birth of a positron-neutrino pair. No wonder the so-called $K$-action capture of an electron from the electron shell of an atom nucleus of an atom in which the electron is also taking place in the transformation of neutrinos inside the nucleus, too, come with a fairly high speeds. (Half-life of most of the isotopes is carried out by $K$-capture, account for about one year). Estimates show that if in the nuclear reaction (1), the time constant of millions of years, in reaction (2) it has a little more than an hour at the same collision frequency particle source "reagent". 
However, if the reaction (1) is needed to encounter two protons, for the nuclear reaction (2) it is required to have not encountered two and three particleselectrons and two protons. The probability of such a three-particle collision in a high-temperature plasma is very low, so the three-particle collision thermonuclear physicists not even consider neglecting them. But chemists no longer ignore the three-particle collisions in the consideration of processes in gases and liquids. Moreover, in many chemical processes (e.g., catalytic) based on the three-particle collisions.

We, too, are not dealing with a high-temperature plasma, and liquid water. And it turns out there are always very specific conditions for the convergence of two protons and electrons. These conditions occur because of thus hydrogen bonds, of which we have already discussed in the previous chapter. Each hydrogen bond linking two adjacent water molecules is generally one proton.

In this case, as shown in the 30 years of the study the structure of water and ice J. R. Bernal and Fowler, a proton is not sitting in the middle of a straight line of communication and a third of the distance from one atom of oxygen to the other.

The proton is not just quietly sitting there, and, as it is later proved by Pauling, then jumps along the connection with a permitted him to another position, located at a distance of one third of the length of the connection from the other oxygen atom of the molecule. The frequency of such jumps is a proton$10^{4} \mathrm{~s}^{-1}$. Twice winner of the Nobel Prize Pauling wittily display this situation the fact that both the proton position said semicircles-"floor proton" statistical model of Pauling.

Similarly, behave on hydrogen bonds heavy water deuterons-nuclei of deuterium atoms.

But sometimes, in the strict world of crystals, and even more in liquid water, with its quasi-crystalline structure misfires happen. And because of one reason or another (fluctuations, shock, or other photon.), proton is knocked out with a hydrogen bond, and is adjacent to. As a result, the latter is just two protons occupying both positions are allowed. These hydrogen bonds are called "orientation-defective". This situation was first described in 1951 by H. Byurrum, and soon a group G. Granihera experimentally confirmed the presence of such defects in ice crystals. Their concentration was in the range of $10^{15}-10^{16} \mathrm{~cm}^{-3}$.

The distance between the two protons (or deuterons in the case of heavy water) in the orientation-defective hydrogen bond is only $0.73 \mathrm{~A}$. In terms of plasma protons converging on such a small distance, it is necessary to heat the plasma to millions of degrees Celsius. It would seem that now the protons in the hydrogen bond are already worthless tunnel to through such a narrow Coulomb barrier separating them to merge in a nuclear reaction (2). A required for this reaction, the electron always has on hand-because it happens among the electron clouds of the atoms of oxygen and hydrogen. Especially, because the protons on a hydrogen bond and then jump along this context, changing places. We can assume that such jumps sometimes lead to a head-on collision of protons too. Then, it would seem that protons have nowhere to go, not to enter into a nuclear reaction (2).

However, such a reaction on the hydrogen bonds of water does not go under normal conditions. The fact is that for its implementation requires orientation, reactive (2) spins of the two protons, parallel to each other, because of the resulting spin deuteron is equal to 1 , and the source of protons back-1/2. The parallel orientation of the spins of the two protons on one of the hydrogen bond is forbidden by the Pauli principle. So here they are antiparallel spin orientation in which the sum of the spins of the proton is equal to zero. To start the nuclear reaction (2), it is required to flip the spin of one of the protons on the defective orientation-hydrogen bond.

This turning back is carried out by means of torsion fields (fields of rotation) that appear when the vortex motion of water in a vortex pipe heat generator of Potapov. About torsion fields we have already 
mentioned in the previous chapter. But here again we note only that but also the phenomenon of changing the direction of the elementary particles of spin torsion fields predicted by the theory of the fields developed by G. I. Spikes already widely used in many technical applications.

When turning the torsion field of spin of one of the protons in the hydrogen bond, there is no violation of the Pauli exclusion principle because the torsion field when it informs the proton, is very small, but the ultimate extra energy. As a result, this proton is on the other, not at the same energy level as the neighbor who does not turn. There is, as physicists say, the splitting of energy levels. Pauli exclusion principle is not been violated, and also costs.

When back at the two protons of orientation-the defective hydrogen bonds are parallel, nothing will prevent these protons to enter into a nuclear reaction (2), leading to the formation of the nuclei of deuterium atoms.

Whirlpools and torsion fields created by them, there are not only in the heat Potapov's generator, but also in countless streams and rivers in the world and in a lot of atmospheric whirlwinds of vapor and water droplets.

Therefore it can be assumed that the deuterium, which is present in the water reservoirs of the earthnot an alien from outer space, and has a terrestrial origin. Now it becomes clear, and the reasons for the differences of impurities of heavy water in different water bodies more than they should be those in which the turbulent flow, rather than calm river.

Further, with respect to the heat source "YUSMAR" of Potapov shown [2, p. 331]:

Discussing the results of the experiments, V. A. Tsarev on cold fusion, implemented using heavy water in the first place is usually considered reaction (17.1) and (17.2) between the deuterons, implying that the heavy water $\mathrm{D}_{2} \mathrm{O}$ has a formula. But hard water obtained by conventional industrial methods by its isolation from natural waters, consists primarily of
$\mathrm{H}_{2} \mathrm{O}$ molecules, as in natural waters in these molecules $10^{4}$ times greater than $\mathrm{D}_{2} \mathrm{O}$ molecules.

Therefore, in such a heavy water deuterium atoms collision with protium atoms occur 104 times more frequently than with deuterium atoms. And when hard water is more diluted and normal, then it reaches the most.

Therefore, we should first consider the following well-known nuclear reaction of the hydrogen cycle:

$$
{ }^{2} \mathrm{D}+{ }^{1} \mathrm{H} \rightarrow{ }^{3} \mathrm{He}+\gamma+5.49 \mathrm{MeV}
$$

Only in this case it can go again at low temperatures resulting in proton collisions with deuterons to defective orientation-hydrogen bonds, which may be deuterons with the same right as the protons.

When the heat generator "YUSMAR" We have recorded an increase in the level of exposure dose of ionizing radiation along the axis of the vortex tube directly behind her steel flange to $15 \mathrm{mR} / \mathrm{hr}$ with natural background $6-8 \mathrm{mR} / \mathrm{hr}$, measured one minute before turning on the heat source.

Just note that the value of this dose is 4 times lower than the maximum allowed $(60 \mathrm{mR} / \mathrm{hr})$, established by the current "Radiation Safety Standards" (RSS-76/87) for the population is not connected to his professional activities with sources of ionizing radiation. For comparison, the natural background of ionizing radiation on the ground in different regions creates dose hovering in the range of 5 to $50 \mathrm{mR} / \mathrm{hr}$, and the world are few places where it is lower than $50 \mathrm{mR} / \mathrm{hr}$.

We have found that ionizing radiation at the heat source has its directivity axis of the vortex tube in the direction of its hot end. This divergence of radiation in the air for the hot end of the tube remains low $\left(<15^{\circ}\right)$. And because the water column length, penetrated by radiation to release into the air is $30 \mathrm{~cm}$, and even 10 $\mathrm{mm}$ steel flange, it is possible to conclude that this really rigid $\gamma$-radiation.

Further, with respect to the heat source of nuclear reactions "YUSMAR" we have [2, p. 334]:

In the previous section we discussed the nuclear reaction (3), going as a result of the two-particle 
collision with a proton in the deuteron orientation-defective water hydrogen bonds. But these relationships can occur and three-particle collision deuteron proton and electron, leading to the following nuclear reaction:

$$
{ }^{2} \mathrm{D}+{ }^{1} \mathrm{H}+\mathrm{e}^{-} \rightarrow{ }^{3} \mathrm{~T}+v_{\mathrm{e}}+5.98 \mathrm{MeV}
$$

The electron in the moment of the proton closer to the deuteron can tunnel through a proton (the nucleus of an ordinary Hydrogen atom-against ${ }^{1} \mathrm{H}$ ), and via ${ }^{2} \mathrm{D}$ deuteron-nucleus of a deuterium atom, which became part of the water molecules instead of against it. Here we turn our attention to the fact that a nuclear reaction (4) none of the physicists never thought.

But this reaction already has no restrictions and does not lead to a violation of the known laws of conservation! It should therefore proceed at a much faster rate than the reaction (3), despite the fact that it happens as a result of three-particle, rather than two-particle collisions.

Reaction (4) are formed ${ }^{3} \mathrm{~T}$ tritium nucleus atoms. And remember, we mentioned in Section 17.2 that researchers around the world for over 10 years, was surprised to note that the cold fusion ${ }^{3} \mathrm{~T}$ output nuclei of tritium atoms somehow by 7-8 orders of magnitude greater than the output of the Helium-3 nuclei of atoms and neutrons. Solve the cause of this no one could.

The researchers believed that the tritium is produced only as a result of the nuclear reaction (4) between the two deuterons. Due to the inertia of thinking, these researchers are accustomed to the whole approach to the yardstick thermonuclear physics neglecting the three-particle collisions, could not think of until a nuclear reaction (4).

Three-particle collisions, as we have noted, really can happen quite often only at relatively low temperatures, and especially on hydrogen bonds in liquid and solids. Namely, in such media, researchers sometimes observed cold fusion.

So big outs when tritium "cold fusion" can be explained by the fact that it is obtained by nuclear reaction (4).

\section{Is a Ten-Year Mystery Finally Solved?}

But being born in the nuclear reaction (4) neutrinos again, as in the case of the reaction (2) takes in the space expanses of the lion's share of the energy of this nuclear reaction, leaving Triton ${ }^{3} \mathrm{~T}$, stuck in water, only small crumbs of this energy transmission it pulse recoil. It deprives us of hope to achieve due to the nuclear reactions (4) additional high heat output in the vortex heat Potapov's generator.

Nevertheless, these results offer hope for use as a heat source in the vortex generator of deuterium and Helium-3 and particularly tritium, with production by other means rather difficult, expensive and dangerous. A Tritium is needed not only for the production of Hydrogen bombs, in recent years, fortunately, losing particular urgency, but also as a raw material in the implementation of controlled thermonuclear fusion, if it still will be at someday implemented and brought to industrial use.

Natural tritium deposits do not exist, because it decays with a half-life of 12 years. At present, tritium needed for the production of Hydrogen bombs, penetrating radiation sources for research, prepared artificially by irradiation of Lithium- 6 isotope neutron flux from a nuclear reactor:

$$
{ }^{6} \mathrm{Li}+\mathrm{n} \rightarrow{ }^{4} \mathrm{He}+{ }^{3} \mathrm{~T}+4.78 \mathrm{MeV}
$$

But reserves of Lithium (and even more of its isotope Lithium-6) in the fields in the world are very limited. Besides, it has extensive use in lithium batteries and the production of other chemical current sources. Thermonuclear physics therefore, it is estimated that only enough Lithium years 100. Sliding the nuclear reaction (4) presents the people in the hands of an inexhaustible source of tritium, which does not require the construction of deep mines for extraction of raw materials. It is necessary for its production according to the reaction (4) water in the world is almost everywhere. 


\section{Laboratory Experiments of N. M. Revinov for Cold Fusion}

N. M. Revinov's installation laboratory in laboratory "HIS" IE "Revinov N. M." in Saran City Karaganda region consists of a transparent container in which it is mounted a tungsten (Wolframium) thin rod that acts as a cathode and the main element of CNF, as the anode-assigned semicircle around the rod inside the vessel thin steel plate. The container half filled with water with a solution of potassium salt (table salt).

Tungsten is a chemical element which has an atomic number 74 in the periodic table D.I. Mendeleev's System Works. Natural tungsten consists of a mixture of five isotopes $\left({ }^{180} \mathrm{~W},{ }^{182} \mathrm{~W},{ }^{183} \mathrm{~W},{ }^{184} \mathrm{~W}\right.$ and ${ }^{186} \mathrm{~W}$ ). In the Revinov's experiment, ${ }^{180}$ Wisotope was used in which nuclear fusion mutates in the isotope ${ }^{184} \mathrm{~W}$. After supplying a current between the cathode and the anode, the process occurs in 1.5-2.0 minutes with a sharp rise in temperature and the formation of spherical shape-plasmoids structure accompanied crackling sound, i.e. passing sonoluminescence effect. Measurements and calculations have shown that ECP (energy conversion rate) turned around 7-8. The experiment itself and instrument performance can be traced in Figs. 1-5. For comparison: in EHH-CTC design of N.M. Revinov this figure is in the range of $15-20$, i.e., the latest installation is twice as effective at heat transfer. As demonstrated above, the EHH-CTC CNF also occurs with the formation of the gas-liquid plasma medium in thermo-camera cells (Figs. 6 and 7). For completeness, Fig. 8 is a structural and functional setup of LVC-RHP construction of N. M. Revinov in IHS laboratory like element base heat generator "YUSMAR" of Yu. S. Potapov, for which true statements and conclusions "Rotational energy" [2], in the sense that the concerns regarding the CNF in these settings (sect. 2). Author's vision view of these problems is discussed in several self-works, particularly in Refs. [3-6].

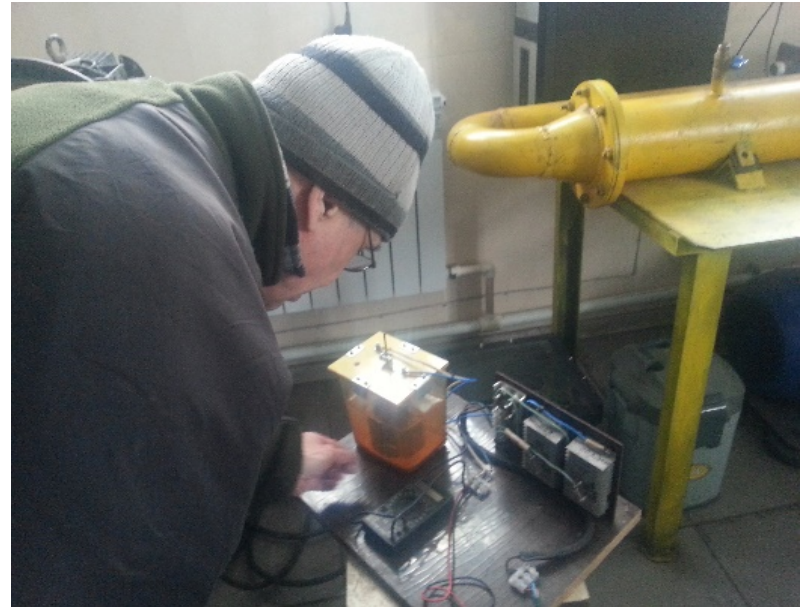

Fig.1 Installing the CNF near the base element of LVC-RHP (installation establishes Nikolai Mikhailovich Revinov).



Fig. 2 Installing the CNF top.

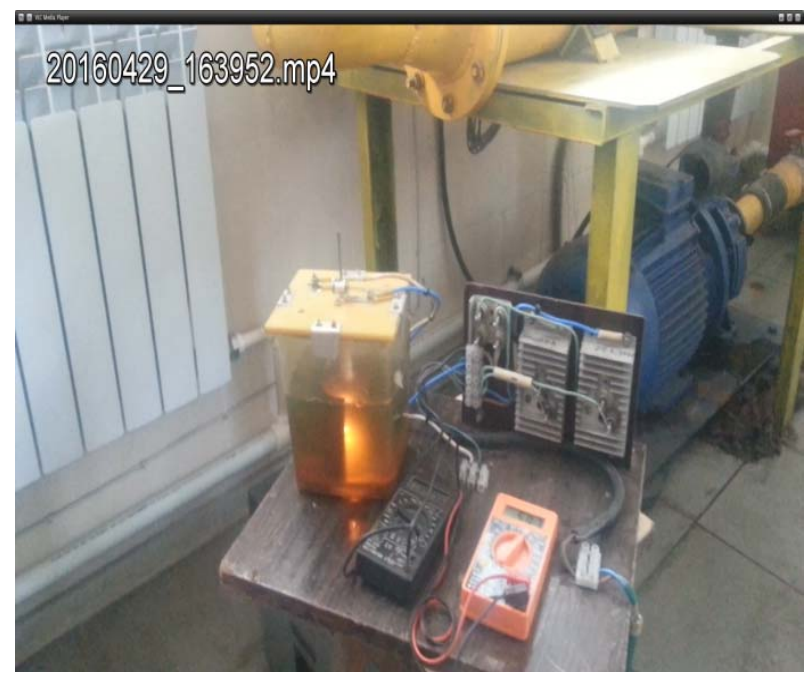

Fig. 3 Installing the CNF has started to work. 


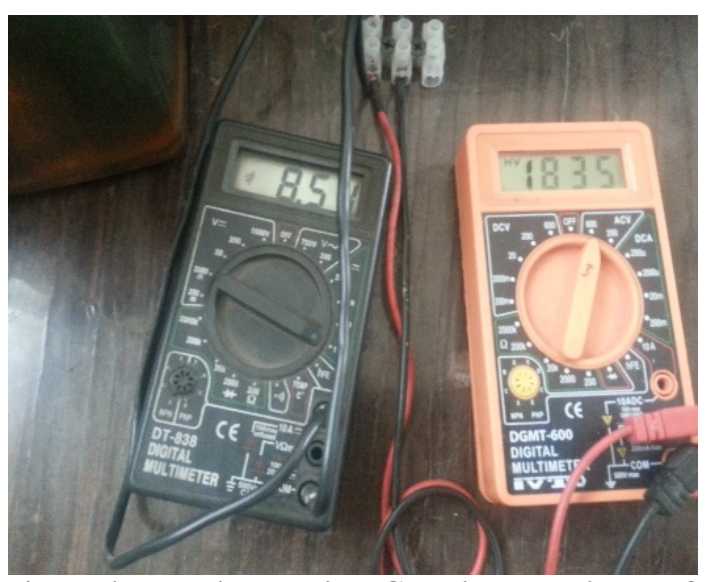

Fig. 4 Indicators installation CNF in the middle of the experiment.



Fig. 5 Setup Indicators CNF in the termination of the experiment.

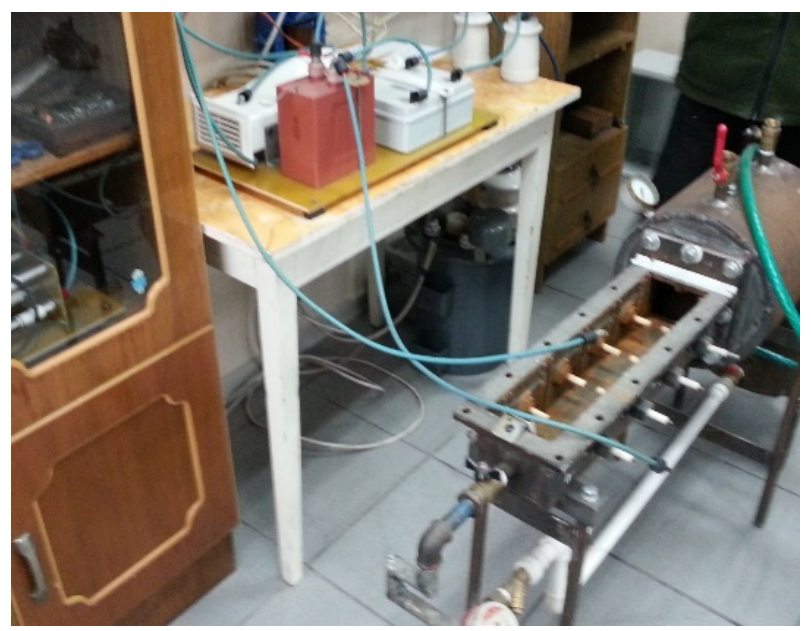

Fig. 6 (a) Installing EHH-CTC in "IHS" laboratory.



Fig. 6 (b) Basic installation EHH-CTC in expanded form: basic setting EHH-CTC with a thermal camera, divided into 4 cells, EDP-electric discharge pulse $\mathrm{HV}$. 




Fig. 7 Building blocks installation EHH-CTC in "IHS" laboratory.

$1-1 \mathrm{~kW}$ transformer, 2-rectifier, 3-the condenser, 4-gas discharge tube.

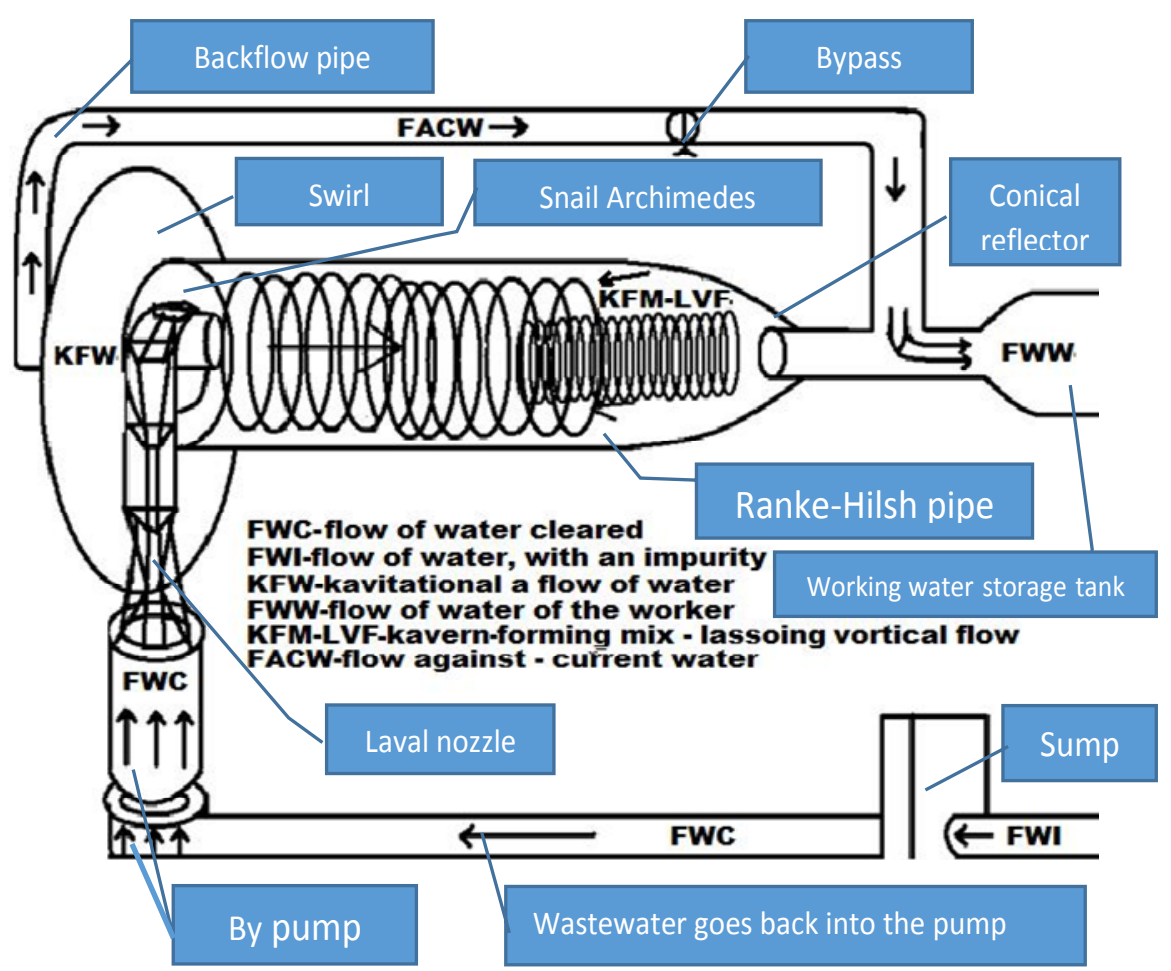

Fig. 8 Structural and functional setup of LVC-RHP.

\section{Conclusion}

This article discusses the problem of replacement of the now existing extensive heating system innovative without fuel intensive heating system. It turns out such a fact that the Ranke-Hilschpipe we have the phenomenon of cold fusion in ambient conditions, so we have to consider in parallel the concept of CNF. The main emphasis is on the ability of cavitation to produce additional energy to produce free heat energy, as well as radiation-nuclear energy. Moreover, this thermal energy is not destructive, but useful.

\section{References}

[1] Vysotsky, V. I., and Kornilova, A. A. 2003. Nuclear Fusion and Transmutation of Isotopes in Biological Systems.

[2] Potapov, Yu. S., and Fominsky, L. P. The Rotational Energy, 389 (Text me shift to Internet format- in plain Word-text format, so pages has more than 286!) Accessed by 
http://www.transgasindustry.com/books/Potapov/vvedeni e.html.

[3] Aubakir, D. A., and Azen, E. 1998. "Transformation into a Useful Source of Energy of a Maxwell's Demon, by Means of Concepts of Chaos, Tornado." In Proceedings of the International Conference Chaos and Structures in Nonlinear Systems. The Theory and Experiment, 233-6.
[4] Aubakir. D. A. 2006. An Innovation-Innovation Is Differed. Semey: Printmaster, 250.

[5] Aubakir, D. A. 2015. Harmony-an Inexhaustible Source of the Being and Eternal Engine of the Intellect, edited by Smirnov, J. Germany: Palmarium, 220.

[6] Aubakir, D. A. 2011. Bases of Systems Theory. Astana: ENU, 500. 